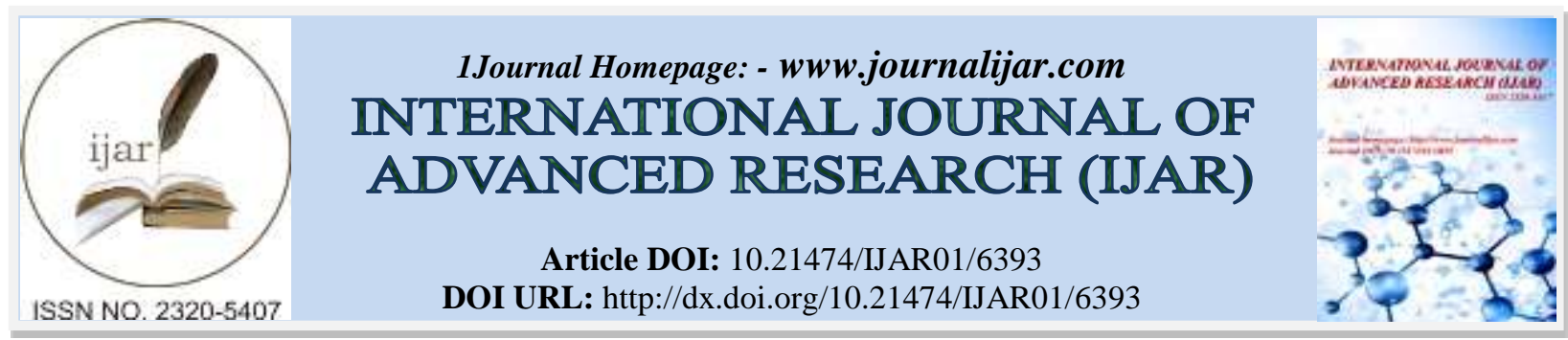

RESEARCH ARTICLE

\title{
THERAPEUTIC APPLICATIONS OF VEGF INHIBITORS IN BREAST CANCER.
}

\author{
Ibrahim Abdullah Al Balawi ${ }^{1}$, Rashid Mir $^{2}$ and Ebtesam Hamoud ${ }^{3}$. \\ 1. Department of surgical oncology, Faculty of Medicine, University of Tabuk, Kingdom of Saudi Arabia. \\ 2. Department of Medical Lab Technology, Faculty of Applied Medical Sciences, University of Tabuk, Kingdom \\ of Saudi Arabia, Tabuk \\ 3. Prince Sultan Oncology Center, King Salman Armed Force Hospital, Tabuk, Saudi Arabia.
}

\section{Manuscript Info}

\section{Manuscript History}

Received: 23 November 2017

Final Accepted: 25 December 2017

Published: January 2018

Key words:-

VEGF receptor 2, Long noncoding RNAs (lncRNAs), SPRY4 intronic transcript 1 (SPRY4-ITI) ,poly(ADPribose) polymerase (PARP), multitargeted receptor tyrosine kinase (RTK).

\begin{abstract}
The importance of angiogenesis in tumour growth and development is well known. Angiogenesis is implicated in the pathogenesis of malignancy and metastasis. The overexpression of vascular endothelial growth factor (VEGF), the key mediator of angiogenesis, is associated with poor prognosis in cancer. Recently the inhibition of angiogenesis has demonstrated clinically significant improvements in outcomes in a variety of malignancies, including breast cancer. As a result, several therapeutic agents that inhibit the actions of VEGF or its receptors are currently in development for use in advanced solid tumours, such breast, colorectal, lung and renal cancer. Antiangiogenesis agents that target the VEGF/VEGF receptor pathway have become an important part of standard therapy in multiple cancer indications. The humanized monoclonal antibody against VEGF, bevacizumab, is the clinically most mature of the antiangiogenic agents and has recently been shown to improve outcome when combined with chemotherapy in the first-line metastatic setting of breast cancer. Different antiangiogenic agents are currently under investigation, including drugs that inhibit the VEGF receptor 2, the cognate receptor for VEGF found on endothelial cells. The combination of antiangiogenic drugs with one another and with other biologic agents is also being explored in an attempt to improve efficacy and to overcome the drug resistance seen with the initial studies of antiangiogenic agents.
\end{abstract}

Copy Right, IJAR, 2018,. All rights reserved.

\section{Introduction:-}

Breast cancer is the most common cancer and the most frequent cause of cancer death among women worldwide. About one in 12 women in the West develop breast cancer at some point in life. It is estimated that 5\%$10 \%$ of all breast cancer cases in women are linked to hereditary susceptibility due to mutations in autosomal dominant genes (1).Breast cancer is a complex, heterogeneous disease classified into hormone-receptor-positive, human epidermal growth factor receptor-2 overexpressing (HER2+) and triple-negative breast cancer (TNBC) based on histological features. Endocrine therapy, the mainstay of treatment for hormone-responsive breast cancer involves use of selective estrogen receptor modulators (SERMs), selective estrogen receptor downregulators (SERDs) and aromatase inhibitors (AIs)(2). Strategies to block ER action, via reduction of estradiol or direct inhibition of ER, have shown major success in the prevention and treatment of breast cancer. Agents that target

Corresponding Author:- Ibrahim Abdullah Al Balawi.

Address:- Department of surgical oncology, Faculty of Medicine, University of Tabuk, Kingdom of 1581 Saudi Arabia. 
estrogen receptor (ER) and HER2 such as tamoxifen and trastuzumab have been the most extensively used therapeutics for breast cancer (3). Crosstalk between ER and other signalling networks as well as epigenetic mechanisms have been envisaged to contribute to endocrine therapy resistance.

Several molecular targets are being explored to target TNBC including androgen receptor, epidermal growth factor receptor (EGFR), poly(ADP-ribose) polymerase (PARP), and vascular endothelial growth factor (VEGF). Receptors, protein tyrosine kinases, phosphatases, proteases, PI3K/Akt signalling pathway, microRNAs (miRs) and long noncoding RNAs (lncRNAs) are potential therapeutic targets (4). The expression of microRNAs (miRNAs), as novel biomarkers, is subject to change in many cancers. Therefore, the overall profile of miRNAs can be used for detection of cancer type, response to therapies, pathological variables, and other factors related to the disease. The recent advances in identification of novel components and regulators of the Microprocessor and Dicer complexes, with particular emphasis on the role of hormone signalling pathways in regulating their activity. It seems that the oncomiRs in the plasma can be an ideal noninvasive candidate biomarker for the early detection of breast cancer and also for following the response of the common therapies. (5).

Understanding hormone regulation of miR production and how this is perturbed in cancer are critical for the development of miR-based therapeutics and biomarkers. miR-based therapeutic approaches include inhibition of oncomiRs by antisense oligonucleotides, restoration of tumour suppressors using miR mimics, and chemical modification of miRs (6).The InRNAs HOTAIR, SPRY4-IT1, GAS5, and PANDAR, new players in tumour development and prognosis may have theranostic applications in breast cancer (7). Long non-coding RNAs (lncRNAs) exert most of their functions through protein interactions. A better understanding of these interactions will facilitate the development of novel therapeutics. The detected lncRNAs are important epigenetic regulators involved in processes such as immune cell regulation and metabolism. Importantly, SPRY4-IT1 exerts important roles in tumor progression and metastasis. The SPRY4 intronic transcript 1 (SPRY4-IT1) is a 708-bp lncRNA on chromosome 5 with a potential functional role in tumorigenesis (8).

The clinical significance of SPRY4-IT1 and the effect of SPRY4-IT1 on cancer progression are unclear. However, detailed molecular mechanisms of SPRY4-IT1 in cancer progression and metastasis are poorly understood. One of the well-known LncRNA HOTAIR is reported to be overexpressed in primary breast cancer and the expression level of HOTAIR is significantly associated with distant metastasis and poor prognosis (9).

Several novel classes of mechanism-based drugs have been designed and synthesised for treatment of breast cancer. Integration of nucleic acid sequencing studies with mass spectrometry-based peptide sequencing and posttranslational modifications as well as rational drug design will provide a more comprehensive understanding of the pathophysiology of breast cancer and help in evolving therapeutic strategies.

\section{Vascular Endothelial Growth Factor (VEGF) Family:-}

VEGF was first identified in guinea pigs, hamsters, and mice by Senger et al. in 1983. (10).It was purified and cloned by Ferrara and Henzel in 1989. (11). VEGF alternative splicing was discovered by Tischer et al. in 1991. (12).Between 1996 and 1997, Christinger and De Vos obtained the crystal structure of VEGF, first at $2.5 \AA$ resolution and later at $1.9 \AA$. Vascular endothelial growth factor (VEGF) not only drives angiogenesis, but also serves as a survival factor for endothelial cells and promotes the abnormal phenotype of blood vessels in tumours (13). Unlike tumour vessels that have VEGF as survival factor, the normal adult vasculature is regarded as largely independent of VEGF for survival, stability, and normal function (14).The VEGF family includes five members;

1. VEGF-A (also referred to as VEGF),

2. VEGF-B,

3. VEGF-C,

4. VEGF-D,

5. VEGF-F,

6. PIGF-placental growth factor (PLGF)-1 and PLGF-2

The factors bind with different affinity and specificity to three tyrosine kinase receptors; vascular endothelial growth factor receptor VEGFR-1, VEGFR-2 . Vascular endothelial cells mainly express VEGFR-1 (binds VEGF-A, VEGF$\mathrm{B}$ and PIGF) and VEGFR-2 (binds predominantly VEGF-A) as depicted in figure 1. The various functions of VEGF family members are depicted in table 1 .VEGFR-3 is mainly found on lymphatic ECs and regulates the lymphangiogenesis .After binding to one receptor, the VEGFs stimulate dimerization of the receptor and initiate a 
signaling cascade that promotes ECs survival, growth and migration.The vessel permeability and endothelial progenitor cells are also stimulated. VEGF-A is the key factor in angiogenesis and primarily stimulates VEGFR-2 (15). VEGF-A is a dimeric disulfide-bound glycoprotein that, due to splicing and posttranslational processing, can be composed of amino acid chains of various lengths. The most common isoform contains 165 amino acids and is overexpressed in several different human tumors (16).

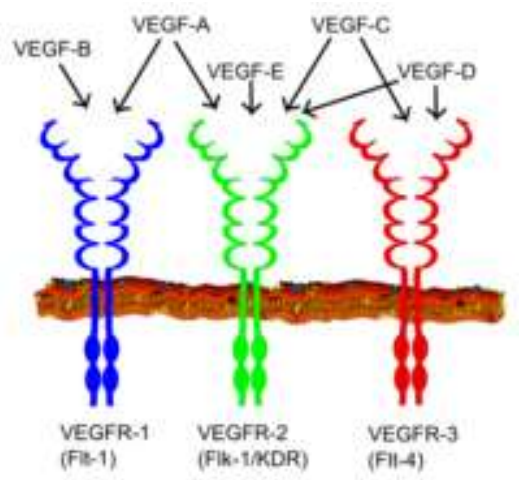

Figure 1:- Types of VEGF and their VEGF receptors.

VEGF is a member of the PDGF/VEGF growth factor family that is often found as a disulfide linked homodimer. It is a glycosylated mitogen that specifically acts on endothelial cells and has various effects, including mediating increased vascular permeability, inducing angiogenesis, vasculogenesis and endothelial cell growth, promoting cell migration, and inhibiting apoptosis (17). VEGFA has multiple isoforms that result from alternative splicing of mRNA from a single, 8-exon VEGFA gene. These are classified into two groups which are referred to according to their terminal exon (exon 8) splice site: the proximal splice site (denoted VEGFxxx) or distal splice site (VEGFxxxb). In addition, alternate splicing of exon 6 and 7 alters their heparin-binding affinity and amino acid number (in humans: VEGF121, VEGF121b, VEGF145, VEGF165, VEGF165b, VEGF189, VEGF206; the rodent orthologs of these proteins contain one fewer amino acids) (18) As depicted in figure 2.

These domains have important functional consequences for the VEGF splice variants, as the terminal (exon 8) splice site determines whether the proteins are pro-angiogenic (proximal splice site, expressed during angiogenesis) or anti-angiogenic (distal splice site, expressed in normal tissues). In addition, inclusion or exclusion of exons 6 and 7 mediate interactions with heparan sulfate proteoglycans (HSPGs) and neuropilin co-receptors on the cell surface, enhancing their ability to bind and activate the VEGF receptors (VEGFRs). Recently, VEGF-C has been shown to be an important inducer of neurogenesis in the murine subventricular zone, without exerting angiogenic effects (19). 


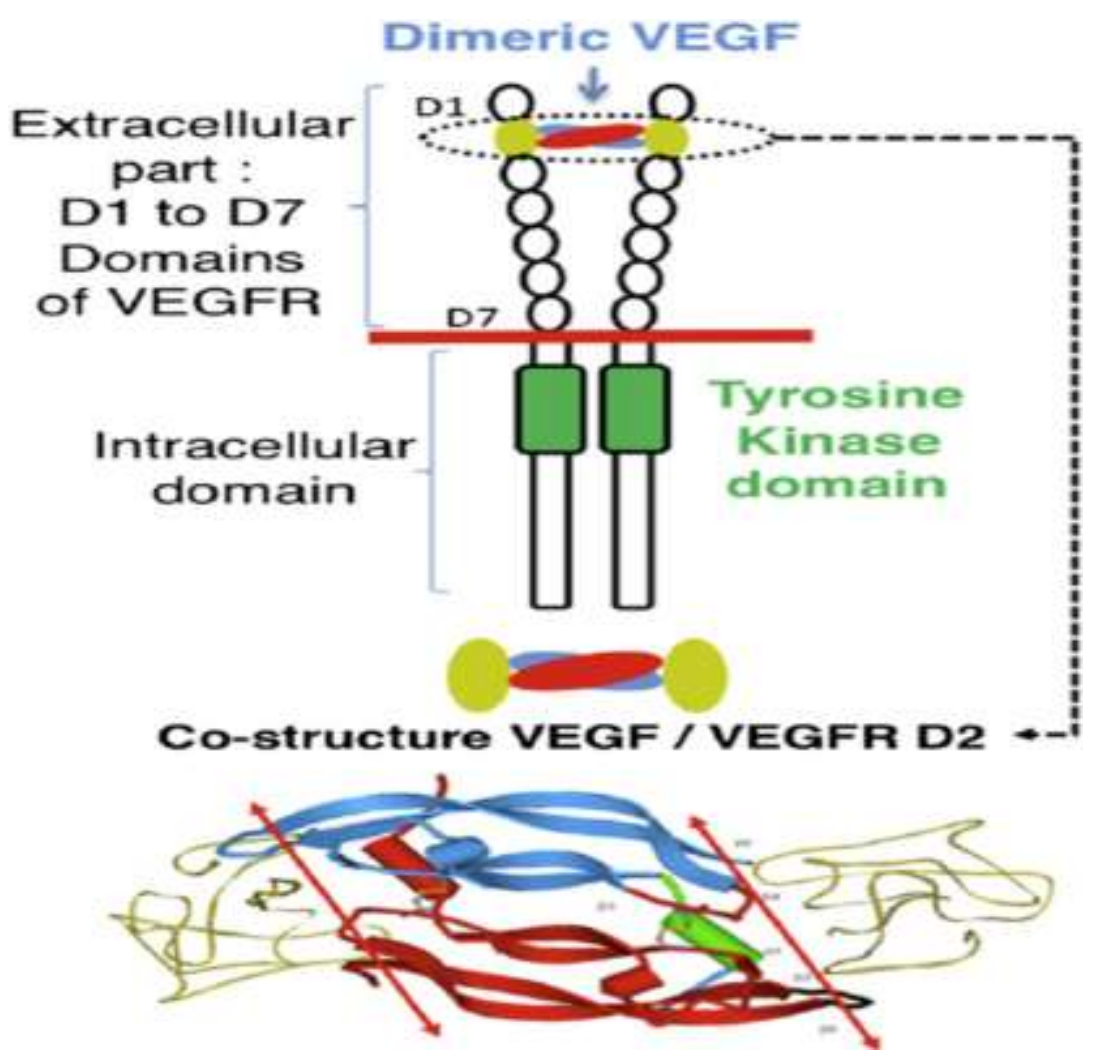

Figure 2:- The VEGF-VEGFR-1 Complex Schematic diagram illustrating our approach for blocking VEGF signaling by inhibition of VEGF-VEGFR PPI. The approach followed in this work does not intend to inhibit the tyrosine kinase site but aims at the inhibition of a PPI (red arrows) through design of a ligand binding to the D2 domain by combining in silico-in vitro screening experiments. See also Figure S1.

Table no 1:- Functions of VEGF family members

\begin{tabular}{|c|c|}
\hline Type & Function \\
\hline VEGF-A & $\begin{array}{l}\text { Initiate Angiogenesis } \\
\uparrow \text { Migration of endothelial cells } \\
\uparrow \text { mitosis of endothelial cells } \\
\uparrow \text { Matrix metalloproteinase activity } \\
\uparrow \alpha v \beta 3 \text { activity } \\
\text { creation of blood vessel lumen } \\
\text { creates fenestrations } \\
\text { Chemotactic for macrophages and granulocytes } \\
\text { Vasodilation (indirectly by NO release) }\end{array}$ \\
\hline VEGF-B & Embryonic angiogenesis (myocardial tissue, to be specific) \\
\hline VEGF-C & Lymphangiogenesis \\
\hline VEGF-D & Needed for the development of lymphatic vasculature surrounding lung bronchioles \\
\hline PIGF & $\begin{array}{l}\text { Important for Vasculogenesis, Also needed for angiogenesis during ischemia, inflammation, wound } \\
\text { healing, and cancer }\end{array}$ \\
\hline
\end{tabular}

The VEGF family comprises from VEGF (VEGF-A), VEGF-B, VEGF-C, VEGF-D, and placental growth factor (PIGF). The VEGF family of receptors consists of three protein-tyrosine kinases. The function of each is mentioned in the table no 1 . Now, the most conventional approach for controlling tumor angiogenesis is blockade of the vascular endothelial growth factor (VEGF) pathway. The results of preclinical studies, substantial therapeutic effects of VEGF blockers have been stated in various types of human cancers, even in progressive or recurrent cancer cases. 


\section{Mechanisms of adverse effects of anti-VEGF therapy for cancer:-}

All members of the VEGF family stimulate cellular responses by binding to tyrosine kinase receptors (the VEGFRs) on the cell surface, causing them to dimerize and become activated through transphosphorylation, although to different sites, times, and extents as depicted in figure 3. The VEGF receptors have an extracellular portion consisting of 7 immunoglobulin-like domains, a single transmembrane spanning region, and an intracellular portion containing a split tyrosine-kinase domain. VEGFR-2 appears to mediate almost all of the known cellular responses to VEGF. The function of VEGFR-1 is less well-defined, although it is thought to modulate VEGFR-2 signaling (20). Another function of VEGFR-1 may be to act as a dummy/decoy receptor, sequestering VEGF from VEGFR-2 binding (this appears to be particularly important during vasculogenesis in the embryo). VEGF-C and VEGF-D, but not VEGF-A, are ligands for a third receptor (VEGFR-3/Flt4), which mediates lymphangiogenesis. The receptor (VEGFR3) is the site of binding of main ligands (VEGFC and VEGFD), which mediates perpetual action and function of ligands on target cells. Vascular endothelial growth factor-C can stimulate lymphangiogenesis (via VEGFR3) and angiogenesis via VEGFR2. Vascular endothelial growth factor-R3 has been detected in lymphatic endothelial cells in CL of many species, cattle, buffalo and primate (21).

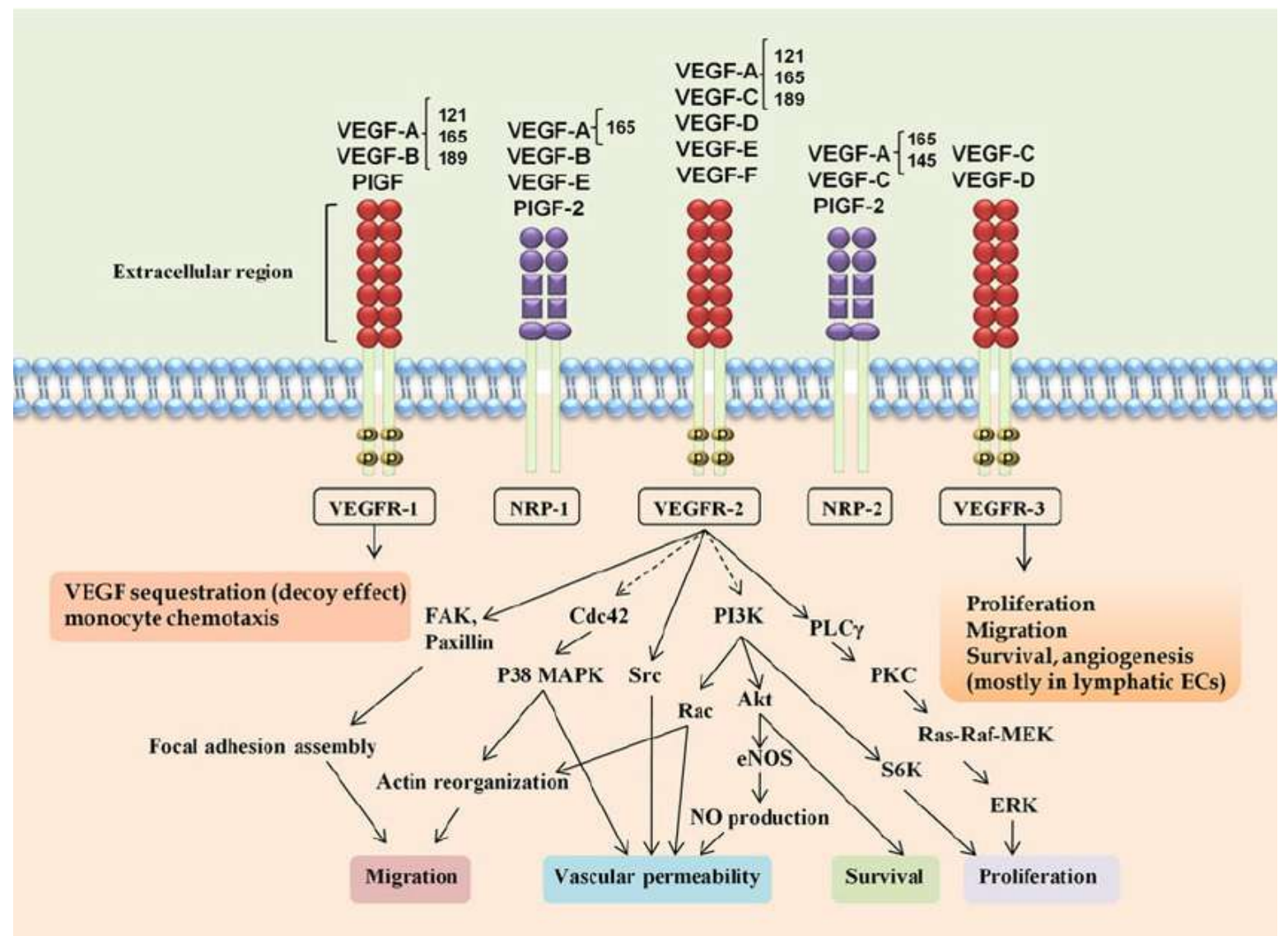

Figure 3:- Action of various VEGF members .

In addition to binding to VEGFRs, VEGF binds to receptor complexes consisting of both neuropilins and VEGFRs. This receptor complex has increased VEGF signalling activity in endothelial cells (blood vessels)(22). Neuropilins (NRP) are pleitrophic receptors and therefore other molecules may interfere with the signalling of the NRP/VEGFR receptor complexes. For example, Class 3 semaphorins compete with VEGF165 for NRP binding and could therefore regulate VEGF-mediated angiogensis. (23) VEGF-A production can be induced in cell that is not receiving enough oxygen. When a cell is deficient in oxygen, it produces HIF, hypoxia-inducible factor, a transcription factor. HIF stimulates the release of VEGF-A, among other functions (including modulation of erythropoiesis). Circulating VEGF-A then binds to VEGF Receptors on endothelial cells, triggering a Tyrosine Kinase Pathway leading to angiogenesis. The expression of angiopoietin-2 in the absence of VEGF leads to endothelial cell death and vascular regression.(23) Conversely, a German study done in vivo found that VEGF concentrations actually decreased after a 25\% reduction in oxygen intake for 30 minutes.(24) HIF1 alpha and HIF1 beta are constantly being produced but 
HIF1 alpha is highly labile, so, in aerobic conditions, it is degraded. When the cell becomes hypoxic, HIF1 alpha persists and the HIF1alpha/beta complex stimulates VEGF release. Breast cancers are heterogeneous in nature, which may account for much of the clinical variability observed from anti-angiogenic therapies. Tumor neovascularization is a complex process that plays a crucial role in the development of several types of cancer (25). The mechanism of hematogenous metastasis requires newly formed capillaries and overexpression of $\geq 1$ positive regulators of angiogenesis such as vascular endothelial growth factor (VEGF) . Previous studies reported the existence of another angiogenic mitogen called endocrine gland-derived VEGF (EG-VEGF), which selectively acts on the endothelium of endocrine gland cells (26). Both angiogenic proteins have been identified in a variety of tissues, and are overexpressed in various cancers (27).Advances in understanding the role of vascular endothelial growth factor (VEGF) in normal physiology are giving insight into the basis of adverse effects attributed to the use of VEGF inhibitors in clinical oncology. These effects are typically downstream consequences of suppression of cellular signalling pathways important in the regulation and maintenance of the microvasculature (28). Downregulation of these pathways in normal organs can lead to vascular disturbances and even regression of blood vessels, which could be intensified by concurrent pathological conditions (29-30). These changes are generally manageable and pose less risk than the tumours being treated, but they highlight the properties shared by tumour vessels and the vasculature of normal organs.

\section{Anti-Vegf Agents In Clinical Use:-}

Advances in understanding the role of vascular endothelial growth factor (VEGF) in normal physiology are giving insight into the basis of adverse effects attributed to the use of VEGF inhibitors in clinical oncology. Now, the most conventional approach for controlling tumor angiogenesis is blockade of the vascular endothelial growth factor (VEGF) pathway. The results of preclinical studies, substantial therapeutic effects of VEGF blockers have been stated in various types of human cancers, even in progressive or recurrent cancer cases. Three drugs, bevacizumab (Avastin ${ }^{\circledR}$ ), sunitinib malate (Sutent ${ }^{\circledR}$, SU11248), and sorafenib (Nexavar ${ }^{\circledR}$, BAY 43-9006) that were developed for antiangiogenic actions have been approved by the United States Food and Drug Administration (FDA) for treatment of patients with specific types of cancer. All three agents inhibit VEGF signalling by blocking VEGF ligand or VEGF receptor function. Sunitinib and sorafenib inhibit platelet-derived growth factor receptors (PDGFR) and some other receptor tyrosine kinases as well.

\section{Bevacizumab:-}

Bevacizumab is the first VEGF inhibitor approved by the FDA for systemic use in cancer. The Bevacizumab is a recombinant humanized monoclonal antibody that blocks angiogenesis by inhibiting vascular endothelial growth factor A (VEGF-A) as depicted in fig 4. Bevacizumab was the first available angiogenesis inhibitor in the United States.

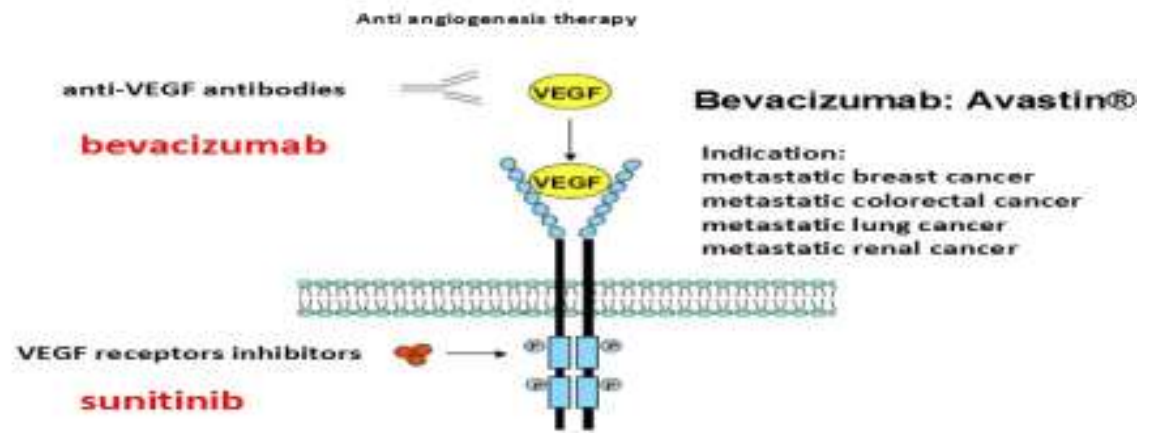

Bevacizumab, sold under the trade name Avastin, is medication used to treat a number of types of cancers and a specific eye disease. For cancer it is given by slow injection into a vein and used for colon cancer, lung cancer, glioblastoma, and renal-cell carcinoma (31). For age-related macular degeneration it is given by injection into the eye. In December 2010, the FDA removed the breast cancer indication from bevacizumab, saying that it had not been shown to be safe and effective in breast cancer patients (32). The combined data from four different clinical trials showed that bevacizumab neither prolonged overall survival nor slowed disease progression sufficiently to outweigh the risk it presents to patients. This only prevented Genentech from marketing bevacizumab for breast cancer (33) On October 11, 2011, the U.S. Food and Drug Administration (FDA) announced that the agency is revoking the agency's approval of the breast cancer indication for bevacizumab after concluding that the drug has not been shown to be safe and effective for that use. 
Bevacizumab is a humanised, function-blocking monoclonal antibody that selectively binds .The anti-VEGF antibody is currently approved in combination with intravenous 5-fluorouracil (5-FU)-based chemotherapy, which typically is a combination of irinotecan, 5-FU, and leucovorin (IFL), for first- or second-line treatment of metastatic carcinoma of the colon or rectum (34-35) and, in conjunction with paclitaxel and carboplatin, for first-line treatment of unresectable, locally advanced, recurrent or metastatic nonsquamous, non-small cell lung cancer (NSCLC) (36). The Bevacizumab also has activity in breast cancer and kidney cancer. Bevacizumab is generally safe and well tolerated but can be accompanied by a variety of adverse effects, which are broadened or intensified by concurrent chemotherapeutic agents (37).

The most common are hypertension, proteinuria, epistaxis, upper respiratory infection, anorexia, stomatitis, diarrhoea or other gastrointestinal symptoms, headache, dyspnea, fatigue, and exfoliative dermatitis. Manifold data have demonstrated that the addition of bevacizumab to chemotherapy improved progression-free survival (PFS), while few trials have revealed its significant overall survival (OS) benefit. Furthermore, it still remains suspended how to maximize the benefits of bevacizumab as first-line therapy for human epidermal growth factor receptor 2 (HER2)-negative breast cancer (38).

\section{Sunitinib:-}

Sunitinib is an small molecule inhibitor of multiple receptor tyrosine kinases implicated in tumour growth, angiogenesis, and metastatic progression. Sunitinib (marketed as Sutent by Pfizer, and previously known as SU11248) is an oral, small-molecule, multi-targeted receptor tyrosine kinase (RTK) inhibitor that was approved by the FDA for the treatment of renal cell carcinoma (RCC) and imatinib-resistant gastrointestinal stromal tumor (GIST) on January 26, 2006. Sunitinib was the first cancer drug simultaneously approved for two different indications.(39)

Sunitinib inhibits cellular signaling by targeting multiple receptor tyrosine kinases (RTKs).These include all receptors for platelet-derived growth factor (PDGF-Rs) and vascular endothelial growth factor receptors (VEGFRs), which play a role in both tumor angiogenesis and tumor cell proliferation. The simultaneous inhibition of these targets therefore reduces tumor vascularization and triggers cancer cell apoptosis and thus results in tumor shrinkage.

Sunitinib also inhibits CD117 (c-KIT), (40) the receptor tyrosine kinase that (when improperly activated by mutation) drives the majority of gastrointestinal stromal cell tumors. It has been recommended as a second-line therapy for patients whose tumors develop mutations in c-KIT that make them resistant to imatinib, or who the cannot tolerate the drug.(41)

In addition, sunitinib binds other receptors. These include:RET ,CD114 and CD135 .The fact that sunitinib targets many different receptors, leads to many of its side effects such as the classic hand-foot syndrome, stomatitis, and other dermatologic toxicities. Clinical trials of patients with anthracycline- and taxane-resistant breast cancer are evaluating sunitinib in combination with taxanes (paclitaxel and docetaxel) in the first-line setting, in combination with capecitabine in the second-line setting, and as a single agent for tumours lacking HER2 receptors, estrogen receptors, and progesterone receptors.(42)

Sunitinib is generally well tolerated. The most common adverse reactions, occurring in more than $20 \%$ of patients, are fatigue, asthenia, diarrhoea, nausea, mucositis/stomatitis, vomiting, dyspepsia, abdominal pain, constipation, hypertension, rash, hand-foot syndrome, skin discolouration, altered taste, anorexia, and mild bleeding.

\section{Sorafenib:-}

Sorafenib (co-developed and co-marketed by Bayer and Onyx Pharmaceuticals as Nexavar), is a kinase inhibitor drug approved for the treatment of primary kidney cancer (advanced renal cell carcinoma), advanced primary liver cancer (hepatocellular carcinoma), and radioactive iodine resistant advanced thyroid carcinoma (43).

Sorafenib is a tyrosine kinase inhibitor and exerts an anti-proliferative and anti-angiogenic activity by blocking the intracellular signal transduction of VEGFR-2 in endothelial cells. Monotherapy using sorafenib has not shown significant results in breast cancer. Sorafenib is an oral, small molecule inhibitor of multiple tyrosine kinase receptors involved both in angiogenesis and tumour cell proliferation: VEGFR-2, VEGFR-3, PDGFR- $\beta$, RAF kinase, FLT3, KIT, p38 MAP kinase (p38-alpha, MAPK14) (43). 
Sorafenib is approved for treatment of advanced renal cell carcinoma and is in phase III clinical trials for hepatocellular carcinoma, metastatic melanoma, and NSCLC. Phase I/II trials of sorafenib plus chemotherapy are ongoing for other solid tumours (44). Side effects associated with sorafenib are mostly mild to moderate, with few severe (Grade 3-4) toxicities. Rash, exfoliative dermatitis, hand-foot skin reaction, diarrhoea, and fatigue are the most common adverse events, occurring in $33-38 \%$ of patients, and are usually Grade 1 or 2 . Mild hypertension, leukopenia, or bleeding is also common (45).

Life-threatening haemorrhage, cardiac ischaemia or infarction, RPLS, and gastrointestinal perforation are uncommon .Therefore, improved glucose handling after systemic inhibition of VEGF signalling probably involves mechanisms other than reduction in islet vascularisation. Consistent changes in glucose handling have not been reported in clinical studies of VEGF inhibitors in cancer patients.

\section{Ramucirumab:-}

Ramucirumab is a humanized monoclonal antibody against the VEGF-binding domain of the VEGFR-2, which is found to be overexpressed in many human breast cancers and seems be an important receptor facilitating VEGFmediated angiogenesis (46).

Ramucirumab binds to VEGF-2 with strong affinity and inhibits interaction between tumor-produced VEGF and VEGFR-2 (47).A phase I study from 2010 examined the effect of ramucirumab against different malignant tumors and revealed promising data for ramucirumab in anti-angiogenic therapy. Keeping in mind that the study population was relatively small and heterogeneous, ramucirumab decreased tumor perfusion and vascularity in $69 \%$ of evaluable patients (48).

Adverse effects associated with ramucirumab are similar to those for bevacizumab. At the moment, a phase III trial on ramucirumab in combination with chemotherapy is ongoing (49). The trial started in 2009 and recruited patients with yet untreated HER2-negative metastatic breast cancer. A phase II open-label study of 141 unresectable patients randomized to receive eribulin monotherapy or eribulin plus ramucirumab in three-week cycles was completed in September 2013. Results of both safety and efficacy await publication (clinicaltrials.gov ID: NCT01427933).

\section{Conclusion:-}

Anti-angiogenic agents impact and reduce the growth of malignant breast tumors. Until now, the use of agents targeting VEGF-driven angiogenesis in combination with standard protocols or in neoadjuvant therapy have shown modest clinical efficacy. A growing number of drugs that inhibit VEGF signalling are being used in the treatment of cancer, commonly in combination with chemotherapy. The efficacy of these agents raises hope for patients with otherwise unresponsive tumours.

\section{Disclosure:-}

The authors report no conflict of interest in this work.

\section{References:-}

1. Sheikh A, Hussain SA, Ghori Q, Naeem N, Fazil A, Giri S, Sathian B, Mainali P,Al Tamimi DM. The spectrum of genetic mutations in breast cancer. Asian Pac J Cancer Prev. 2015;16(6):2177-85.

2. Kleibl Z, Kristensen $V N$ Women at high risk of breast cancer: Molecular characteristics, clinical presentation and management.. Breast. 2016 Aug; 28:136-44.

3. Kota K, Brufsky A, Oesterreich S, Lee A. Estradiol as a Targeted, Late-Line Therapy in Metastatic Breast Cancer with Estrogen Receptor Amplification. Cureus.2017 Jul 6;9(7):e1434.

4. Nagini S. Breast Cancer: Current Molecular Therapeutic Targets and New Players. Anticancer Agents Med Chem. 2017;17(2):152-163.

5. Farsinejad S, Rahaie M, Alizadeh AM, Mir-Derikvand M, Gheisary Z, Nosrati H,Khalighfard S. Expression of the circulating and the tissue microRNAs after surgery, chemotherapy, and radiotherapy in mice mammary tumor. Tumour Biol. 2016 Oct;37(10):14225-14234.

6. Fletcher CE, Dart DA, Bevan CL. Interplay between steroid signalling and microRNAs: implications for hormone-dependent cancers. Endocr Relat Cancer. 2014 Oct;21(5):R409-29. 
7. Sang Y, Tang J, Li S, Li L, Tang X, Cheng C, Luo Y, Qian X, Deng LM, Liu L, Lv XB. LncRNA PANDAR regulates the G1/S transition of breast cancer cells by suppressing p16(INK4A) expression. Sci Rep. 2016 Mar $1 ; 6: 22366$

8. Li J, Chen Y, Chen Z, He A, Xie H, Zhang Q, Cai Z, Liu Y, Huang W. SPRY4-IT1: A novel oncogenic long non-coding RNA in human cancers. Tumour Biol. 2017 Jun;39(6):1010428317711406.

9. Gupta R. A. et al. Long non-coding RNA HOTAIR reprograms chromatin state to promote cancer metastasis. Nature 464, 1071-1076, 10.1038/nature08975 (2010).

10. Senger, DR; Galli, SJ; Dvorak, AM; Perruzzi, CA; Harvey, VS; Dvorak, HF (25 February 1983). "Tumor cells secrete a vascular permeability factor that promotes accumulation of ascites fluid.". Science. 219 (4587): 983-5

11. Ferrara, N; Henzel, WJ (15 June 1989). "Pituitary follicular cells secrete a novel heparin-binding growth factor specific for vascular endothelial cells.". Biochemical and Biophysical Research Communications. 161 (2): 8518

12. Tischer, E; Mitchell, R; Hartman, T; Silva, M; Gospodarowicz, D; Fiddes, JC; Abraham, JA (25 June 1991). "The human gene for vascular endothelial growth factor. Multiple protein forms are encoded through alternative exon splicing.". The Journal of Biological Chemistry. 266 (18): 11947-54

13. Nishida N, Yano H, Nishida T, Kamura T, Kojiro M .Angiogenesis in cancer.Vasc Health Risk Manag. 2006; 2(3):213-9.

14. Longo R, Sarmiento R, Fanelli M, Capaccetti B, Gattuso D, Gasparini G. Anti-angiogenic therapy: rationale, challenges and clinical studies. Angiogenesis. 2002;5:237-256.

15. Mancuso MR, Davis R, Norberg SM, O'Brien S, Sennino B, Nakahara T, Yao VJ, Inai T, Brooks P, Freimark B, Shalinsky DR, Hu-Lowe DD, McDonald DM. Rapid vascular regrowth in tumors after reversal of VEGF inhibition. J Clin Invest. 2006;116:2610-2621

16. Niu G., Chen X. Vascular endothelial growth factor as an anti-angiogenic target for cancer therapy. Curr. Drug Targets. 2010;11:1000-1017.

17. Grothey A., Ellis L.M. Targeting angiogenesis driven by vascular endothelial growth factors using antibodybased therapies. Cancer J. 2008;14:170-177

18. Cébe Suarez, S; Pieren, M; Cariolato, L; Arn, S; Hoffmann, U; Bogucki, A; Manlius, C; Wood, J; BallmerHofer, K. "A VEGF-A splice variant defective for heparan sulfate and neuropilin-1 binding shows attenuated signaling through VEGFR-2". Cellular and Molecular Life Sciences. 63: 2067-2077

19. Shin, Y. J.; Choi, J. S.; et al. (2010). "Induction of vascular endothelial growth factor receptor-3 mRNA in glial cells following focal cerebral ischemia in rats". J Neuroimmunol. 229 (1-2): 81-90.

20. LeCouter J, Lin R, Ferrara N.The role of EG-VEGF in the regulation of angiogenesis in endocrine glands. Cold Spring Harb Symp Quant Biol. 2002; 67():217-21.

21. Keramidas M, Faudot C, Cibiel A, Feige JJ, Thomas M.Mitogenic functions of endocrine gland-derived vascular endothelial growth factor and Bombina variegata 8 on steroidogenic adrenocortical cells.J Endocrinol. 2008 Mar; 196(3):473-82.

22. Holmes, Katherine; Roberts, Owain Ll; Thomas, Angharad M.; Cross, Michael J. (2007). "Vascular endothelial growth factor receptor-2: Structure, function, intracellular signalling and therapeutic inhibition". Cellular Signalling. 19 (10): 2003-12.

23. Herzog, B; Pellet-Many, C; Britton, G; Hartzoulakis, B; Zachary, I. C. (2011). "VEGF binding to NRP1 is essential for VEGF stimulation of endothelial cell migration, complex formation between NRP1 and VEGFR2, and signaling via FAK Tyr407 phosphorylation". Molecular Biology of the Cell. 22(15): 2766-2776

24. Oltmanns, K. M.; Gehring, H; Rudolf, S; Schultes, B; Hackenberg, C; Schweiger, U; Born, J; Fehm, H. L.; Peters, A (2006). "Acute hypoxia decreases plasma VEGF concentration in healthy humans". AJP: Endocrinology and Metabolism. 290(3): E434-9.

25. Schlessinger, J. and Lemmon, M. A. Sh2 And Ptb domains in tyrosine kinase signaling. Sci. STKE 2003 191, RE12.

26. Wiesmann, C., Muller, Y. A., and de Vos, A. M. Ligand-binding sites in Ig-like domains of receptor tyrosine kinases. J. Mol. Med. 2000, 78, 247-260.

27. Blechman, J. M., Lev, S., Brizzi, M. F., Leitner, O., Pegoraro, L., Givol, D., and Yarden, Y. Soluble c-kit proteins and antireceptor monoclonal antibodies confine the binding site of the stem cell factor. J. Biol. Chem. 1993, 268, 4399-4406. 47.

28. Blechman, J. M. and Yarden, Y. Structural aspects of receptor dimerization. c-kit as an example. Ann. N Y Acad. Sci. 1995, 766, 344-362. 
29. Herren, B., Rooney, B., Weyer, K. A., Iberg, N., Schmid, G., and Pech, M. (1993) Dimerization of extracellular domains of platelet-derived growth factor receptors. A revised model of receptor-ligand interaction. J. Biol. Chem. 268, 15088-15095. 49.

30. Fuh, G., Li, B., Crowley, C., Cunningham, B., and Wells, J. A. (1998) Requirements for binding and signaling of the kinase domain receptor for vascular endothelial growth factor. J. Biol. Chem. 273, 11197-11204

31. Manzo A, Montanino A, Carillio G, Costanzo R, Sandomenico C, Normanno N,Piccirillo MC, Daniele G, Perrone F, Rocco G, Morabito A. Angiogenesis Inhibitors in NSCLC. Int J Mol Sci. 2017 Sep 21;18(10). pii: E2021.

32. Jennifer Couzin-Frankel; Yasmin Ogale (8 July 2011). "Once on 'Fast Track', Avastin Now Derailed". Science. 333 (6039): 143-4.

33. Larsen FO, Markussen A, Diness LV, Nielsen D. Efficacy and Safety of Capecitabine, Irinotecan, Gemcitabine, and Bevacizumab as Second-Line Treatment in Advanced Biliary Tract Cancer: A Phase II Study. Oncology. 2017 Sep 21

34. Ntavatzikos A, Spathis A, Patapis P, Machairas N, Peros G, Konstantoudakis S, Leventakou D, Panayiotides IG, Karakitsos P, Koumarianou A. Integrating TYMS,KRAS and BRAF testing in patients with metastatic colorectal cancer. World J Gastroenterol. 2017 Aug 28;23(32):5913-5924.

35. Hurwitz H, Fehrenbacher L, Novotny W, Cartwright T, Hainsworth J, Heim W, Berlin J, Baron A, Griffing S, Holmgren E, Ferrara N, Fyfe G, Rogers B, Ross R, Kabbinavar F. Bevacizumab plus irinotecan, fluorouracil, and leucovorin for metastatic colorectal cancer. N Engl J Med. 2004;350:2335-2342

36. Sandler A, Gray R, Perry MC, Brahmer J, Schiller JH, Dowlati A, Lilenbaum R, Johnson DH. Paclitaxelcarboplatin alone or with bevacizumab for non-small-cell lung cancer. N Engl J Med. 2006;355:2542-2550.

37. Gordon MS, Cunningham D. Managing patients treated with bevacizumab combination therapy. Oncology. 2005;69 Suppl 3:25-33.

38. Li C1, Xiang A2, Chen X3, Yin K4, Lu J4, Yin W5,6.Optimizing the treatment of bevacizumab as first-line therapy for human epidermal growth factor receptor 2 (HER2)-negative advanced breast cancer: an updated meta-analysis of published randomized trials. Onco Targets Ther. 2017 Jun 27;10:3155-3168.

39. US Food and Drug Administration (2006). "FDA approves new treatment for gastrointestinal and kidney cancer".

40. Hartmann JT, Kanz L (November 2008). "Sunitinib and periodic hair depigmentation due to temporary c-KIT inhibition". Arch Dermatol. 144 (11): 1525-6.

41. Quek R, George S (February 2009). "Gastrointestinal stromal tumor: a clinical overview". Hematol. Oncol. Clin. North Am. 23 (1): 69-78

42. "Prescribing information for Sutent (sunitinib malate)". Pfizer, Inc, New York NY.

43. Gradishar W.J. Sorafenib in locally advanced or metastatic breast cancer. Expert Opin. Investig. Drugs. 2012;21:1177-1191.

44. Schwartzberg L.S., Tauer K.W., Hermann R.C., Makari-Judson G., Isaacs C., Beck J.T., Kaklamani V., Stepanski E.J., Rugo H.S., Wang W., et al. Sorafenib or placebo with either gemcitabine or capecitabine in patients with her-2-negative advanced breast cancer that progressed during or after bevacizumab. Clin. Cancer Res. 2013;19:2745-2754.

45. Keating GM, Santoro A (2009). "Sorafenib: a review of its use in advanced hepatocellular carcinoma". Drugs. 69 (2): 223-40

46. Wilhelm SM, Adnane L, Newell P, Villanueva A, Llovet JM, Lynch M (October 2008). "Preclinical overview of sorafenib, a multikinase inhibitor that targets both Raf and VEGF and PDGF receptor tyrosine kinase signaling". Mol. Cancer Ther. 7 (10): 3129-40.

47. Choueiri T.K., Mayer E.L., Je Y., Rosenberg J.E., Nguyen P.L., Azzi G.R., Bellmunt J., Burstein H.J., Schutz F.A. Congestive heart failure risk in patients with breast cancer treated with bevacizumab. J. Clin. Oncol. 2011;29:632-638.

48. Spratlin J.L., Cohen R.B., Eadens M., Gore L., Camidge D.R., Diab S., Leong S., O’Bryant C., Chow L.Q., Serkova N.J., et al. Phase I pharmacologic and biologic study of ramucirumab (imc-1121b), a fully human immunoglobulin g1 monoclonal antibody targeting the vascular endothelial growth factor receptor-2. J. Clin. Oncol. 2010;28:780-787.

49. Phase III Study of Docetaxel + Ramucirumab or Placebo in Breast Cancer. [(accessed on 1 December 2014)]; Available online: http://clinicaltrials.gov/ct2/show/NCT00703326. 